\title{
A many-body lubrication model
}

\section{Bertrand MAURY}

Department of Mathematics, University of Houston, Houston, Texas 77204 USA.

\begin{abstract}
In [2], we presented direct numerical simulations of the motion of solid particles in a viscous fluid. In such simulations, numerical problems are likely to occur when particles get very close to each other: the mesh is to be refined in the gap zone, which is computationally expensive. In order to overcome this problem, we introduce here a lubrication model to handle those "near collisions". The objective is to couple this new approach with the global computation of the flow. Nevertheless, we will concentrate in the present paper on the lubrication model only. Indeed, the physical situation we will be dealing with is the motion of a close-packed arrangement of particles in a fluid, such that the influence of the fluid on the global behavior is restricted to lubrication forces.
\end{abstract}

\section{Un modèle de lubrification pour un grand nombre de particules}

Résumé. Dans une précédente note [2], nous avons présenté des simulations directes du mouvement d'un ensemble de particules baignant dans un fluide visqueux. Le problème majeur lié à ce type de simulation tient au fait que les particles peuvent être très proches les unes des autres : le maillage doit alors être raffiné, ce qui peut augmenter considérablement le temps de calcul. Nous présentons ici un modèle spécifique pour le traitement de ces interactions rapprochées entre particules. Bien que le but final soit de coupler cette approche avec le calcul de l'écoulement dans son ensemble, nous nous contenterons ici de présenter uniquement le modèle de lubrification. La situation physique que nous étudions correspond ainsi à une collection assez dense de particules pesantes baignant dans un fluide dont l'action sur les particules se limite aux forces de lubrification.

\section{Version française abrégée}

On s'intéresse au mouvement d'un ensemble de particules pesantes dans une cavité, sous l'action de la pesanteur et des forces d'interaction à courte distance, liées à la présence d'un fluide visqueux dans les zones interstitielles. Le modèle utilisé est basé sur un développement asymptotique de l'interaction entre deux sphères en mouvement relatif, en fonction de $\varepsilon$, distance entre elles (voir [1]). Nous généralisons ce modèle au cas de $N$ 
particules, ce qui conduit à un système de $3 N$ équations différentielles non-linéaires. Nous montrons que ce système peut être écrit de façon compacte (équation (9)) dans l'espace de configuration associé au problème, espace de dimension $3 N$. De cette formulation peut être déduit un système différentiel vérifié par les quantités sensibles: les distances entre les particules, qui interviennent de façon singulière dans l'expression des forces de lubrification. En explicitant certains termes dans chacune des équations composant ce système, on se ramène à un ensemble d'équations différentielles toujours non linéaires, mais indépendantes.

Le schema global proposé est basé sur un découplage du calcul des distances (estimées par résolution des équations différentielles scalaires de type (14)), et la résolution du mouvement des corps eux même (système linéaire symétrique (16)).

La stabilité et la précision de l'algorithme proposé tiennent aux deux points suivants :

1. Les distances, susceptibles de tendre vers 0 , sont calculées à chaque itération par résolution d'une équation différentielle qu'il est peu coûteux de résoudre avec une grande précision, et tout en s'assurant que la distance reste strictement positive.

2. Lors du calcul des vitesses des particules (résolution du système (16)), les forces de lubrification sont implicites en vitesse, de telle sorte que la vitesse relative de deux particules proches sera fortement pénalisée.

A titre d'illustration, nous présentons la simulation numérique du mouvement de 4000 particules de formes et de tailles différentes s'écoulant entre deux parois sous l'action de la pesanteur dans un domaine périodique dans la direction verticale.

1. Introduction, notations. We consider a set of $2 \mathrm{D}$ rigid bodies in a cavity. The torque-force couple acting on each body is supposed to be restricted to the sum of a prescribed body force, such as gravity, and the action exerted by the other particles and the walls of the container.

The only interaction we will take into account corresponds to the lubrication phenomenon: when two moving surfaces are close to each other, a Poiseuille-type flow develops in the intersurface gap, leading to high local stress values. The action of the lubrication forces is dissipative: the force opposes to the relative motion, and there is no force if the bodies are steady. The model we will use is a extension of the following one: when two spheres are approaching with a relative velocity $\mathbf{u}$, distance $d$, the modulus of the force acting normally on both surfaces can be estimated, when the inter-sphere distance $d$ is small, by

$$
|\mathbf{F}|=\mu \frac{|\dot{\mathbf{u}}|}{d} .
$$

The force $\mathbf{F}$ is directed along the line joining the two centers, and is opposed to the motion of each surface. The same way, a shearing motion $\mathbf{u}^{\perp}$ induces a force of modulus

$$
\left|\mathbf{F}^{\perp}\right|=\mu^{\perp}|\dot{\mathbf{u}}| \ln \left(\frac{d_{o}}{d}\right)
$$

which is orthogonal to $\mathbf{F}$. The parameters $\mu, \mu^{\perp}$, and $d_{o}$ depend on the viscosity of the lubricating fluid, and on both radii of the spheres. As a matter of fact, a shearing motion 
also creates a force along $\mathbf{F}$, but this force, usually negligible compared to $\mathbf{F}$, will not be taken into account in our analysis. Since the lubrication forces depend in a singular way on the distance between the surfaces, a numerical method based on a straightforward time discretization is not suitable for two reasons:

- As two bodies get close to each other, the time step will have to be highly reduced, even if the other parts of the system would not require such a time step reduction,

- The distance between two bodies appears to be a very sensitive parameter, because of the stiffness of the lubrication forces with respect to it. Therefore, it must not be numerically estimated as a difference between two finite quantities.

A set of $N$ rigid bodies in the plane can be described by a $3 N$ vector

$$
X=\left(\mathbf{x}_{1}, \theta_{1}, \ldots, \mathbf{x}_{N}, \theta_{N}\right),
$$

where $\mathbf{x}_{i}$ is the mass center of the body $i$, and $\theta_{i}$ a rotation angle. We denote by $\Omega_{i}\left(\mathbf{x}_{i}, \theta_{i}\right)$ the moving $\mathrm{i}^{\text {th }}$ body, and by $\Omega_{0}$ the exterior domain delimiting the cavity (see figure 1 ). For $i, j$ in $[0, N]$, let us now introduce $\mathrm{D}_{i j}\left(\Omega_{i}, \Omega_{j}\right)=\mathrm{D}_{i j}\left(\mathbf{x}_{i}, \theta_{i}, \mathbf{x}_{j}, \theta_{j}\right)$, the distance between the two bodies. We denote by $\mathbf{C}_{i}^{j}$ (resp. $\mathbf{C}_{j}^{i}$ ) the point of $\partial \Omega_{i}\left(\right.$ resp. $\partial \Omega_{j}$ ) at which the distance is reached (see figure 1).

$\Omega_{\mathrm{o}}$

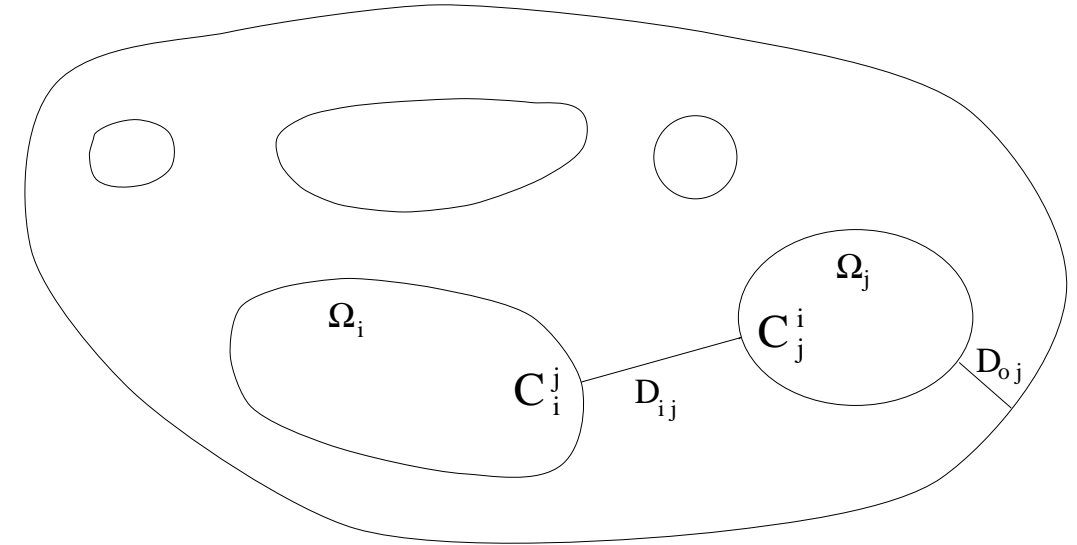

Fig. IV.1 : Notations.

We introduce the family of constrained configuration spaces

$$
S_{\varepsilon}=\left\{\left(\mathbf{x}_{i}, \theta_{i}\right)_{1 \leq i \leq N} \in \mathbb{R}^{3 N} \text { s.t. } \mathrm{D}_{i j}>\varepsilon, \forall(i, j) \in[0, N]^{2}, i \neq j\right\} .
$$

The natural space for our problem is then $S_{o}$. The non-overlapping condition can be expressed by $X \in \overline{S_{o}}$.

Remark : In computations, bodies might overlap. It will be necessary to extend the definition of $\mathrm{D}_{i j}$ outside $S_{o}$. We will use the fact that there exists a smooth extension of $\mathrm{D}_{i j}$ in the neighborhood of the boundary of $S_{o}$, which takes negative values as soon as bodies $i$ and $j$ overlap. The points $\mathbf{C}_{i}^{j}$ and $\mathbf{C}_{j}^{i}$ are still defined, and their dependance on $\left(\mathbf{x}_{i}, \theta_{i}, \mathbf{x}_{j}, \theta_{j}\right)$ is smooth. 
2. Lubrication model. In [1], Kim and Karrila give estimates for the forces induced by the relative motion of two spheres in term of $\varepsilon$, the distance between them. Let us consider two bodies $\Omega_{i}$ and $\Omega_{j}$ and their corresponding points $\mathbf{C}_{i}^{j}$ and $\mathbf{C}_{j}^{i}$ (see figure 1 ). A natural way to generalize this lubrication model between two spheres is the following: $\dot{\mathbf{C}}_{i}^{j}$ and $\dot{\mathbf{C}}_{j}^{i}$ being the velocities of the material points $\mathbf{C}_{i}^{j}$ and $\mathbf{C}_{j}^{i}$, the lubrication phenomenon leads to 2 opposite forces $\mathbf{F}_{i}^{j}$ and $\mathbf{F}_{j}^{i}$ acting on $\mathbf{C}_{i}^{j}$ and $\mathbf{C}_{j}^{i}$, respectively. According to [1], these forces can be estimated by

$$
\mathbf{F}_{i}^{j}=-\mathbf{F}_{j}^{i}=-\kappa\left(\mathrm{D}_{i j}\right)\left[\left(\dot{\mathbf{C}}_{i}^{j}-\dot{\mathbf{C}}_{j}^{i}\right) \cdot \mathbf{e}_{i j}\right] \mathbf{e}_{i j}-\kappa^{\perp}\left(\mathrm{D}_{i j}\right)\left[\left(\dot{\mathbf{C}}_{i}^{j}-\dot{\mathbf{C}}_{j}^{i}\right) \cdot \mathbf{e}_{i j}^{\perp}\right] \mathbf{e}_{i j}^{\perp},
$$

which can be written

$$
\mathbf{F}_{i}^{j}=\left[-\kappa\left(\mathrm{D}_{i j}\right) \mathbf{e}_{i j} \otimes \mathbf{e}_{i j}-\kappa^{\perp}\left(\mathrm{D}_{i j}\right) \mathbf{e}_{i j}^{\perp} \otimes \mathbf{e}_{i j}^{\perp}\right] \cdot\left(\dot{\mathbf{C}}_{i}^{j}-\dot{\mathbf{C}}_{j}^{i}\right)
$$

The vector $\mathbf{e}_{i j}$ is the unit vector along $\mathbf{C}_{i}^{j} \mathbf{C}_{j}^{i}$, and $\mathbf{e}_{i j}^{\perp}$ is orthogonal to $\mathbf{e}_{i j}$. The functions $\kappa$ and $\kappa^{\perp}$ take the following form

$$
\kappa(d)=\mu \frac{1}{d}, \quad \kappa^{\perp}(d)=\mu^{\perp} \ln \left(d_{o} / d\right),
$$

where $\mu$ and $\mu^{\perp}$ depend only on the geometry (local radii of curvature) and on the viscosity of the lubricating fluid.

As a matter of fact, a shearing motion also creates a force along $\mathbf{e}_{i j}$, so that the complete model should also contain a term in $\mathbf{e}_{i j} \otimes \mathbf{e}_{i j}^{\perp}$. As this force is usually negligible compared to $\kappa\left(\mathrm{D}_{i j}\right) \mathbf{e}_{i j} \otimes \mathbf{e}_{i j}$, it will not be taken into account in the approach presented here. Furthermore, we will suppose that $\kappa$ and $\kappa^{\perp}$ are given functions which are asymptotically equivalent to the expressions given by (7), and vanish when $d$ is greater than a given value $d_{o}$. In the numerical application, $d_{o}$ will be taken equal to the characteristic size of the bodies. For the sake of simplicity, as a first approximation of the behaviour of the system, $\mu$ and $\mu^{\perp}$ will be taken constant. The underlying physical assumptions are that the bodies have a similar shape, which is close to a circle, and that the viscosity of the fluid is constant.

3. Global system. We denote by $m_{i}$ the mass of the body $i$, by $I_{i}$ its moment of inertia, and by $\boldsymbol{\Phi}_{i}$ the body force acting on it. The components of the vector $X=\left(\mathbf{x}_{i}, \theta_{i}\right)_{1 \leq i \leq N}$ verify the system of ordinary differential equations (with $\mathbf{F}_{i}^{j}$ given by (6)):

$$
m_{i} \ddot{\mathbf{x}}_{i}=\boldsymbol{\Phi}_{i}+\sum_{j \neq i} \mathbf{F}_{i}^{j}\left(\dot{\mathbf{C}}_{i}^{j}, \dot{\mathbf{C}}_{j}^{i}\right), \quad I_{i} \ddot{\theta}_{i}=\sum_{j \neq i} \mathbf{x}_{i} \mathbf{C}_{i}^{j} \times \mathbf{F}_{i}^{j}\left(\dot{\mathbf{C}}_{i}^{j}, \dot{\mathbf{C}}_{j}^{i}\right)
$$

Let us now introduce a new function $\mathrm{D}_{i j}^{\perp}$, defined in the neighborhood of $X$ as the projection of $\mathbf{C}_{i}^{j} \mathbf{C}_{j}^{i}$ onto $\mathbf{e}_{i j}^{\perp}$, where $\mathbf{e}_{i j}^{\perp}$ is set to its value at $X$, and $\mathbf{C}_{i}^{j}$ and $\mathbf{C}_{j}^{i}$ are the material points associated to $\mathbf{C}_{i}^{j}(X)$ and $\mathbf{C}_{j}^{i}(X)$. We denote the gradient of $\mathrm{D}_{i j}$ by $\mathbf{G}_{i j}$, and the gradient of $\mathrm{D}_{i j}^{\perp}$ by $\mathbf{G}_{i j}^{\perp}$. Both $\mathrm{D}_{i j}$ and $\mathrm{D}_{i j}^{\perp}$ are considered functions of $X$, so that $\mathbf{G}_{i j}$ and $\mathbf{G}_{i j}^{\perp}$ are $3 \mathrm{~N}-$ vectors. 
Proposition 1 The interaction forces can be expressed in terms of $\mathrm{D}_{i j}, \mathbf{G}_{i j}, \mathbf{G}_{i j}^{\perp}, \kappa\left(\mathrm{D}_{i j}\right)$, $\kappa^{\perp}\left(\mathrm{D}_{i j}\right)$, and the velocity vector $\dot{X}$. More precisely, the system (8) can be written in the following form

$$
\mathrm{M} \ddot{X}=\Phi-\frac{1}{2} \sum_{i \neq j}\left[\kappa\left(\mathrm{D}_{i j}\right) \mathbf{G}_{i j} \otimes \mathbf{G}_{i j}+\kappa^{\perp}\left(\mathrm{D}_{i j}\right) \mathbf{G}_{i j}^{\perp} \otimes \mathbf{G}_{i j}^{\perp}\right] \cdot \dot{X},
$$

where $\mathrm{M}$ is the mass matrix: $\mathrm{M}=\operatorname{diag}\left(m_{1}, m_{1}, I_{1}, \ldots \ldots, m_{N}, m_{N}, I_{N}\right)$.

This is a straightforward calculation based on the exact expressions of $\mathbf{G}_{i j}$ and $\mathbf{G}_{i j} \frac{\perp}{\text { : }}$

$$
\begin{aligned}
& \mathbf{G}_{i j}=\left(\ldots, 0,-\mathbf{e}_{i j},-\mathbf{x}_{i} \mathbf{C}_{i}^{j} \times \mathbf{e}_{i j}, 0, \ldots, 0, \mathbf{e}_{i j}, \mathbf{x}_{j} \mathbf{C}_{j}^{i} \times \mathbf{e}_{i j}, 0, \ldots\right), \\
& \mathbf{G}_{i j}^{\perp}=\left(\ldots, 0,-\mathbf{e}_{i j}^{\perp},-\mathbf{x}_{i} \mathbf{C}_{i}^{j} \times \mathbf{e}_{i j}^{\perp}, 0, \ldots, 0, \mathbf{e}_{i j}^{\perp}, \mathbf{x}_{j} \mathbf{C}_{j}^{i} \times \mathbf{e}_{i j}^{\perp}, 0, \ldots\right) .
\end{aligned}
$$

Remark : The velocity $\mathbf{V}=\dot{X}$ verifies the associated energy equation

$$
\frac{d}{d t}\left(\frac{1}{2} \mathrm{MV} \cdot \mathbf{V}\right)-\Phi \cdot \mathbf{V}+\Psi(\mathbf{V}, \mathbf{V})=0
$$

where $\Psi$ is the diffusion symmetric non-negative bilinear form which appears in (9). The quantity $\Psi(\mathbf{V}, \mathbf{V})$ is the rate of dissipated energy, $\mathbf{M V} \cdot \mathbf{V} / 2$ the kinetic energy, and $\Phi \cdot \mathbf{V}$ the power of the body forces. If external forces $\Phi$ are limited to gravity, $-\Phi \cdot \mathbf{V}$ is the derivative of the potential energy with respect to time. In the case where the "bounding box" is the whole plane $\left(\Omega_{o}=\emptyset\right), \Psi$ is never definite, even if the functions $\kappa$ and $\kappa^{\perp}$ are supported by $\mathbb{R}^{+}$, for any rigid motion of the whole set of bodies does not dissipate energy (such a motion is orthogonal to both $\mathbf{G}_{i j}$ and $\mathbf{G}_{i j}^{\perp}$ ).

4. Equation for $D_{p q}$. The basis of our approach is to work directly on the quantities $\mathrm{D}_{p q}$. To that purpose, an equation verified by each of the distances is established. One has

$$
\dot{\mathrm{D}}_{p q}=\mathbf{G}_{p q} \cdot \dot{X}, \quad \ddot{\mathrm{D}}_{p q}=\mathbf{G}_{p q} \cdot \ddot{X}+\dot{\mathbf{G}}_{p q} \cdot \dot{X},
$$

so that, using (9),

$$
\begin{aligned}
\ddot{\mathrm{D}}_{p q}= & \mathbf{G}_{p q} \cdot \mathrm{M}^{-1} \Phi-\mu \frac{\dot{\mathrm{D}}_{p q}}{\mathrm{D}_{p q}} \mathbf{G}_{p q} \cdot \mathrm{M}^{-1} \mathbf{G}_{p q} \\
& -\frac{1}{2} \sum_{i \neq j} \kappa\left(\mathrm{D}_{i j}\right)\left(\mathbf{G}_{i j} \cdot \mathbf{V}\right)\left(\mathbf{G}_{p q} \cdot \mathrm{M}^{-1} \mathbf{G}_{i j}\right) \\
& -\frac{1}{2} \sum_{i \neq j} \kappa^{\perp}\left(\mathrm{D}_{i j}\right)\left(\mathbf{G}_{i j}^{\perp} \cdot \mathbf{V}\right)\left(\mathbf{G}_{p q} \cdot \mathrm{M}^{-1} \mathbf{G}_{i j}^{\perp}\right)+\dot{\mathbf{G}_{p q}} \cdot \dot{X} .
\end{aligned}
$$

5. Time discretization scheme. We denote the time step by $k$, its reciprocal by $\alpha$. The scheme that we propose to solve the original problem is based on a decoupling of the locations of the particles and the distances between them. The non-linear system of 
ODE's corresponding to equations (13) is transformed into a set of independent ODE's by expliciting $\mathbf{V}$, the $\mathbf{G}_{i j}$ 's, and the $\mathrm{D}_{i j}$ 's for $(i, j) \neq(p, q)$. The obtained equations have the form

$$
\ddot{a}=k_{o}-k_{1} \frac{\dot{a}}{a}, a(0)=a_{o}, \dot{a}(0)=a_{1} .
$$

We denote by $K\left(k_{o}, k_{1}, a_{o}, a_{1}, t\right)$ an approximation of $a(t)$, obtained by any numerical method suitable for this kind of ODE. The global scheme is then:

$$
\begin{array}{rll}
\mathrm{D}_{p q}^{m+1} & = & K\left(k_{o}, k_{1}, a_{o}, a_{1}, k\right), \\
\left(\alpha \mathrm{M}+A^{m}\right) \mathbf{V}^{m+1} & = & \alpha \mathbf{M V}^{m}+\Phi \\
X^{m+1} & = & X^{m}+k \mathbf{V}^{m+1} \\
\mathbf{G}_{i j}^{m+1}, \mathbf{G}_{i j}^{\perp m+1} & \text { estimated at } & X^{m+1} \text { according to }(10), 0 \leq i<j \leq N,
\end{array}
$$

where $A^{m}$ is the stiffness matrix

$$
A^{m}=\frac{1}{2} \sum_{i \neq j}\left(\kappa\left(D_{i j}^{m+1}\right) \mathbf{G}_{i j}^{m} \otimes \mathbf{G}_{i j}^{m}+\kappa^{\perp}\left(\mathrm{D}_{i j}^{m+1}\right) \mathbf{G}_{i j}^{\perp m} \otimes \mathbf{G}_{i j}^{\perp m}\right) .
$$

and the symbols $k_{o}, k_{1}, a_{o}$, and $a_{1}$ are given explicitely by

$$
\begin{gathered}
k_{o}=\mathbf{G}_{p q}^{m} \cdot \mathrm{M}^{-1} \Phi-\frac{1}{2} \sum_{(i, j) \neq(p, q)} \kappa\left(\mathrm{D}_{i j}^{m}\right)\left(\mathbf{G}_{i j}^{m} \cdot \mathbf{V}^{m}\right)\left(\mathbf{G}_{p q}^{m-1} \cdot \mathrm{M}^{-1} \mathbf{G}_{i j}^{m}\right) \\
+\alpha\left(\mathbf{G}_{p q}^{m}-\mathbf{G}_{p q}^{m-1}\right) \cdot \mathbf{V}^{m}-\frac{1}{2} \sum_{i \neq j} \kappa^{\perp}\left(\mathrm{D}_{i j}^{m}\right)\left(\mathbf{G}_{i j}^{\perp m} \cdot \mathbf{V}^{m}\right)\left(\mathbf{G}_{p q}^{m} \cdot \mathrm{M}^{-1} \mathbf{G}_{i j}^{\perp m}\right) \\
k_{1}=\mu \mathbf{G}_{p q}^{m} \cdot \mathrm{M}^{-1} \mathbf{G}_{p q}^{m}, \quad a_{o}=\mathrm{D}_{p q}^{m}, \quad a_{1}=\mathbf{G}_{p q}^{m} \cdot \mathbf{V}^{m}
\end{gathered}
$$

Remark : If the time step is too large, the computed $X$ might exit

$$
S_{o}=\left\{X, \mathrm{D}_{i j}(X)>0, \forall i \neq j\right\}
$$

which means that some particles overlap. Since the small quantities and the locations of the particles are decoupled, such a behavior of the computation does not necessarily prevent us from getting an accurate estimate of the inter-particle distances. Nevertheless, in order to keep working on realistic configurations, close particles are artificially kept at a fixed small distance $\varepsilon$. The last operation, which is performed at each time step of the computation, can be seen as a projection onto $S_{\varepsilon}=\left\{X, \mathrm{D}_{i j}(X)>\varepsilon, \forall i \neq j\right\}$. We are not yet able to define properly this mapping as a projection, nor to estimate the possible loss of accuracy caused by this operation.

6. Numerical solution, results. Equation (14), which corresponds to the step (15) of the scheme, is integrated once with respect to time, and the obtained first order ODE is solved by a $4^{\text {th }}$ order Runge-Kutta scheme. The system (16) is solved by a conjugate gradient preconditioned by the diagonal. Noteworthily, the s.p.d. matrix $\alpha \mathrm{M}+A^{m}$ presents similarities with a discrete Laplace operator on a non-uniform mesh, the $\mathrm{D}_{i j}$ distribution playing the role of the local mesh step size. The step (18) is the most time-consuming. We developed an algorithm to compute those gradients for the case of any convex shape. It will be described in a next paper. 
Remark : The distance between two bodies does not have to be estimated with high accuracy. As soon as this distance drops below a certain value (size of the interval supporting $\kappa$ and $\kappa^{\perp}$ ), the corresponding quantity $\mathrm{D}_{i j}$ is activated, and its evolution is solved with accuracy.

We present the simulation of 4000 particles of different sizes and shapes colliding in a domain periodic in the vertical direction. The body forces are restricted to gravity. The ratio horizontal dimension / average size of the bodies is about 35, and the solid fraction is 0.4. Figures 2 and 3 represent the initial (random) configuration and the configuration at time $t=1.6$ (500 iterations), respectively. The latter presents cavitation zones which developed during the computation. In figure 4, for each particle, we plotted its vertical velocity versus the horizontal coordinate of its mass center. It gives an illustration of the Laplace-type behavior of the operator we introduced.

\section{References}

[1] S. Kim S. and S. J. Karrila, Microhydrodynamics: Principles and Selected Applications, Butterworth-Heinemann, Boston, 1991.

[2] B. Maury and R. Glowinski, Fluid-Particle Flow: a Symmetric Formulation, C. R. Acad. Sci. Paris, t. 324, Série I, pp. 1079-1084, 1997. 


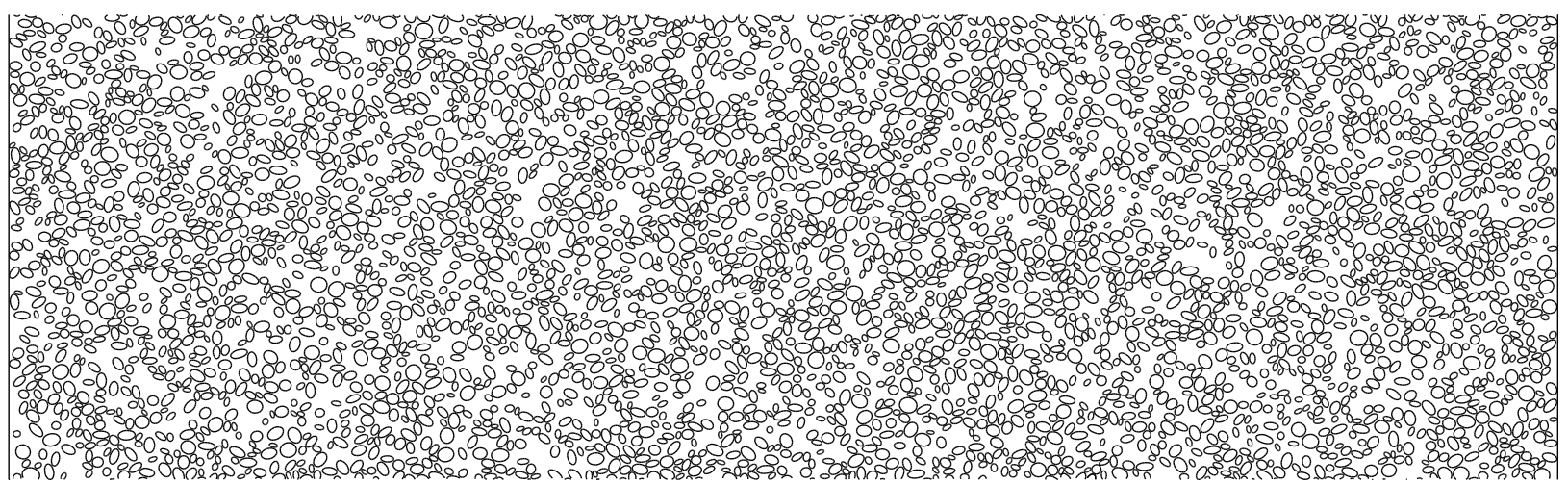

Fig. IV.2 : Initial configuration. Configuration initiale.

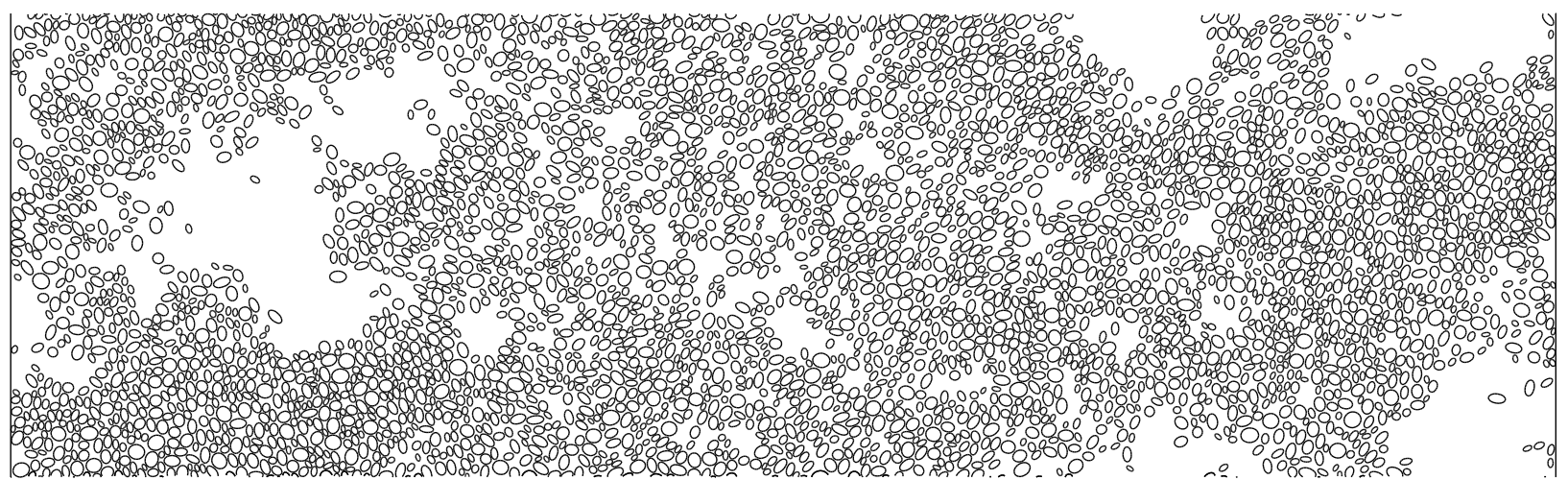

Fig. IV.3 : Configuration at time $t=1.6$. Configuration au temps $t=1.6$.

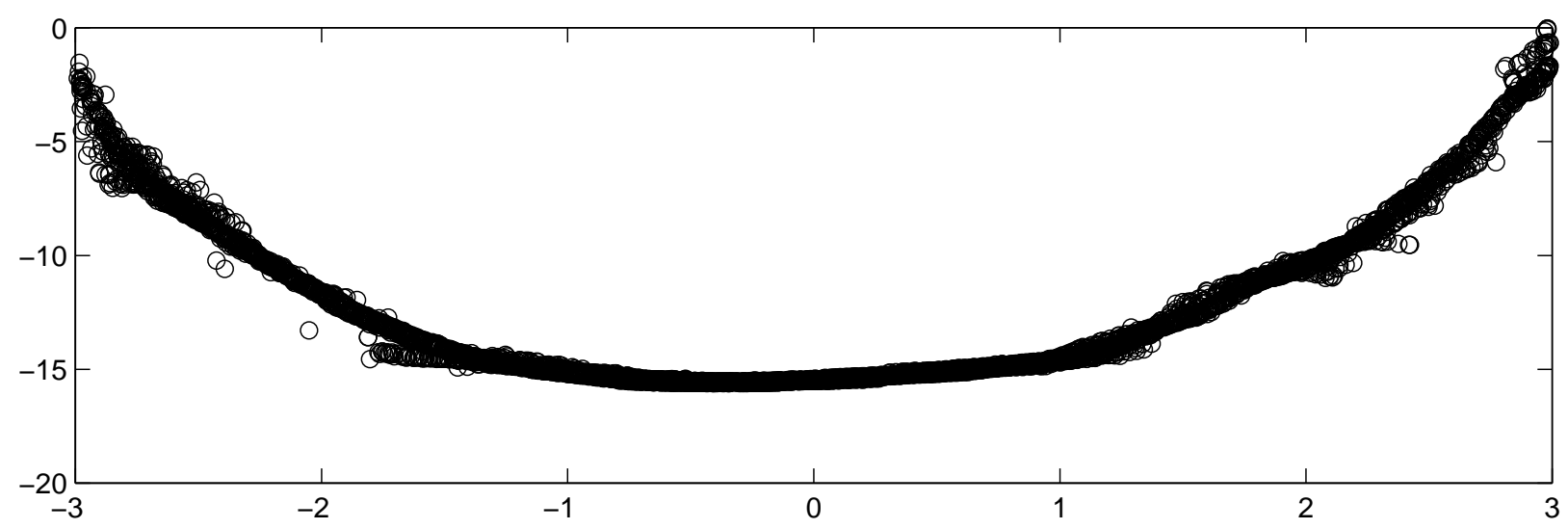

Fig. IV. 4 : Vertical velocities at $t=1.6$. Vitesses verticales $\grave{a} t=1.6$. 